\title{
Prognostic Significance of TMPRSS2-ERG Fusion Gene in Prostate Cancer
}

\author{
VLASTIMIL KULDA ${ }^{1}$, ONDREJ TOPOLCAN ${ }^{2}$, RADEK KUCERA ${ }^{2}$, MICHAELA KRIPNEROVA ${ }^{3}$, \\ KRISTYNA SRBECKA ${ }^{1}$, MILAN HORA ${ }^{4}$, ONDREJ HES ${ }^{5}$, JIRI KLECKA ${ }^{6}$, \\ VACLAV BABUSKA ${ }^{1}$, MILENA ROUSAROVA ${ }^{2}$, VERONIKA BENSON $^{7}$ and MARTIN PESTA ${ }^{3}$ \\ Departments of ${ }^{1}$ Medical Chemistry and Biochemistry, ${ }^{3}$ Biology, ${ }^{4}$ Urology and ${ }^{5}$ Pathology, \\ Faculty of Medicine in Pilsen, Charles University in Prague, Pilsen, Czech Republic; \\ ${ }^{2}$ Department of Nuclear Medicine - Immunoanalytic Laboratory, \\ University Hospital in Pilsen, Pilsen, Czech Republic; \\ ${ }^{6}$ Urocentrum Plzen, Pilsen, Czech Republic; \\ ${ }^{7}$ Institute of Microbiology, v.v.i., Academy of Sciences of the Czech Republic, Prague, Czech Republic
}

\begin{abstract}
Background/Aim: Current research of prostate cancer (PCa) offers a promising way of identifying patients with adverse prognosis who do benefit from radical treatment that can affect quality of life as resections are associated with numerous side-effects. The aim of our study was to evaluate the relationship of TMPRSS2-ERG fusion gene status, tumor tissue prostate-specific antigen (PSA), prostate cancer antigen 3 (PCA3), miR-23b, miR-26a and miR-221 expression levels in combination with preoperative serum PSA level to the risk of PCa recurrence after radical prostatectomy. Patients and Methods: The study group consisted of 108 patients who underwent radical prostatectomy. PSA was measured in peripheral blood collected preoperativelly. The expression of TMPRSS2-ERG transcript and the expression of miR-23b, miR-26a and miR221 in formalin-fixed, paraffin-embedded (FFPE) tumor tissues was analyzed by reverse transcription $(R T)$ real-time polymerase chain reaction (PCR). Results: Significantly shorter time to recurrence was observed in patients with high expression of TMPRSS2-ERG $(p=0.0020)$. High levels of preoperative PSA $(>10.0 \mathrm{ng} / \mathrm{ml})$ proved to be marker of shorter time to recurrence $(p=0.0153)$. The most promising marker of the risk of recurrence after radical prostatectomy was a combination of high level of preoperative serum PSA
\end{abstract}

Correspondence to: Dr. Martin Pesta, Department of Biology, Faculty of Medicine in Pilsen, Alej Svobody 76, 326 00, Plzen, Czech Republic. Tel: +420 377593261, Fax: +420 377593209, e-mail: martin.pesta@lfp.cuni.cz

Key Words: Prostate cancer, TMPRSS2-ERG, PSA, PCA3, microRNA, prognosis. and high expression of TMPRSS2-ERG fusion transcript in tumor tissue ( $p=0.0001)$. Conclusion: A combination of high preoperative serum PSA and high expression of TMPRSS2ERG could be promising in distinguishing those tumors that are aggressive and life-threatening .

Prostate cancer (PCa) belongs to the most commonly diagnosed cancers worldwide having a higher incidence in Western countries, which might be due to environmental and lifestyle factors, as well as the greater scale of prostatespecific antigen (PSA) screening in the developed countries. $\mathrm{PCa}$ has been proven to be a very heterogenous disease, with individual cases differing in both the speed of progression and overall prognosis (1). The main issue of PCa management is to distinguish those tumors that are subsequently progressing. This would make it possible to limit the number of patients undergoing radical surgery to cases of life-threatening tumors as radical prostatectomy is associated with numerous sideeffects $(2,3)$. To assure radical treatment in cases of aggressive prostate cancer a set of markers reliably indicating the nature of the individual case needs to be identified.

Current research offers a promising way of identifying patients with adverse prognosis. It is based on the knowledge of the pathogenesis of PCa and on the role of the fusion gene TMPRSS2-ERG described and linked to PCa by Tomlin et al. in 2005 (4). The origin of the TMPRSS2-ERG oncogene can be traced to a recurrent rearrangement (translocation, interstitial deletion) on long arm of chromosome 21 that fuses androgen-regulated prostate-specific gene promoter of the transmembrane protease serine 2 (TMPRSS2), locus 21q22.3, and the gene $E R G$, locus 21q22.2, a member of the transcription factor erytroblastosis virus E26 transforming sequence family (ETS) (5). Prostate tissue has a strong 
androgen dependency and so does $\mathrm{PCa}$, with the signal pathways being mediated by androgen receptors (6). As a result of a TMPRSS2-ERG fusion, overexpression of oncogenic transcription factor ERG is driven by androgens through androgen-responsive elements of TMPRSS2 5'untranslated region. Nevertheless, as the disease evolves into castration-resistant $\mathrm{PCa}, T M P R S S 2-E R G$ fusion gene overcomes androgen regulation (7). Fusion gene TMPRSS2$E R G$ appears in early high grade prostatic intraepithelial neoplasia (PIN) and it has been observed that its presence in $\mathrm{PCa}$ cells promotes the loss of the tumor supressor gene PTEN and other alterations, which in turn lead to the speed up of progression of the disease (8).

In addition to analysis of TMPRSS2-ERG fusion gene significance, we also focused on selected microRNAs. MicroRNAs are non-coding RNAs consisting of a small number of nucleotides ( $22 \mathrm{nt})$ that can influence gene expression by interfering with translation or by destabilising target mRNAs. In this way, microRNAs can affect cancerogenesis in both directions; slow the progression by inbibiting oncogen expression or fasten it by downregulating tumor supressors (9). Many studies have described changes in expression of microRNAs in PCa tissue and their involvement in initiation, progression and the effects of treatment (10). Based on our research of published literature, we chose three tumor-suppressive miRNAs (miR-23b, miR26a and miR-221) whose levels could be of interest in making prediction of tumor aggressiveness.

The expression level of miR-23b has been observed to be down-regulated in $\mathrm{PCa}$ and restoration of the expression inhibited cancer cell proliferation, migration and invasion in $\mathrm{PCa}$ cell lines (11). In PCa, miR-23b supresses protooncogene Src kinase and its up-regulation shows a strong positive correlation to better prognosis (12). Both miR-26a and miR-221 function as cell proliferation inhibitors, miR26a inhibits prostate cancer progression by repression of Wnt5a (13) and La-related protein 1 (LARP1) (14). Lower miR-221 expression was associated with a higher risk of recurrence after radical prostatectomy (15).

Furthermore, we included analysis of tumor tissue expression of PSA and prostate cancer antigen 3 (PCA3), formerly referred to as differential display code 3 (DD3). PCA3 is a long non-coding RNA (lncRNA) beginning to be used as a diagnostic marker of PCa. Recent meta-analysis concluded that urine PCA3 test had acceptable sensitivity and specificity for the diagnosis of PCa (16). There are studies showing association of high PCA3 expression with pathological features of PCa, being predictive of high Gleason score, high-stage and high-volume disease $(17,18)$. However, less is known about prognostic significance of PCA3 on the basis of the follow-up of patients.

The aim of our study was to evaluate the relationship of TMPRSS2-ERG fusion gene status, tumor tissue PSA, PCA3,
miR-23b, miR-26a and miR-221 expression levels in combination with routinely used preoperative serum PSA level to the risk of PCa recurrence after radical prostatectomy and, in this way, to predict the aggressivity of the tumor.

\section{Patients and Methods}

Patients. Our study group consisted of 108 patients who underwent radical prostatectomy between January 2011 and June 2012 at the Department of Urology of the University Hospital in Pilsen, Czech Republic. All the patients exhibited elevated serum PSA levels or abnormal digital rectal examination. Indication for surgery was confirmed by positive biopsy results, namely the detection of cancerous cells. The median age was 62.9 years (range=43.8-73.9). Clinicopathological data, such as age at the time of surgery, preoperative PSA level, stage of the disease according to the International Union against Cancer (IUCC) and Gleason score are listed in Table I. Approval was obtained from the Institutional Ethics Committee and written informed consent from each patient.

Tissue samples and RNA extraction. The FFPE (formalin-fixed, paraffin-embedded) tissue samples were used for RNA extraction as we described previously $(19,20)$. Briefly, areas selected for analysis highlighted by pathologist (either tumor tissue or adjacent normal prostate tissue) were manually macrodisected from $15-\mu \mathrm{m}$ thick FFPE tissue sections prepared with microtome (Leica RM 2135; Leica Biosystems, Nussloch, Germany). Total RNA was isolated using the RNeasy FFPE Kit (Qiagen, GmbH, Hilden, Germany) according to the protocol of the manufacturer. The concentration of the isolated RNA was measured with NanoDrop ND-1000 spectrophotometer (Thermo Fisher Scientific, Wilmington, DE, USA); in case of lower concentration than $15 \mathrm{ng} / \mu \mathrm{l}$ the isolation was repeated.

Quantitative estimation of TMPRSS2-ERG, PSA and PCA3 expression. Reverse transcription (RT) was performed from $250 \mathrm{ng}$ of total RNA with Superscript III Reverse Transcriptase (Life Technologies, Carlsbad, CA, USA) and random hexamers as primers. Quantitative estimation of TMPRSS2-ERG, PSA and PCA3 transcripts was performed by real-time polymerase chain reaction (PCR) method with UPL probes (Universal ProbeLibrary; Roche, Mannheim, Germany) on Stratagene Mx3005P apparatus (Agilent Technologies, Santa Clara, CA, USA). The sequences of primers for TMPRSS2-ERG fusion gene (forward primer 5'-TAGGC GCGAGCTAAGCAG-3' targeting TMPRSS2 exon 1 and reverse primer 5'-GTCCATAGTCGCTGGAGGAG-3' targeting ERG exon 4) were previously designed and validated by other researchers $(21$, 22). We found the appropriate UPL probe (probe \#1) for this pair of primers by ProbeFinder Software (Roche, Mannheim, Germany). The sequences of primers and corresponding UPL probes for PSA mRNA (forward primer 5'-GTGCTTGTGG CCTCTCGT-3', reverse primer 5'-CAGCAAGATCACGCTTTTGT-3', probe \#44) and $P C A 3$ lncRNA (forward primer 5'-TGGGAAGGACCTGAT GATACA-3', reverse primer 5'-TGTGTGGCCTCAGA TGGTAA3', probe \#66) were generated by ProbeFinder Software. The PCR reactions were carried out in 96-well plates in a volume of $20 \mu \mathrm{l}$ containing $1.0 \mu \mathrm{l}$ of RT product, $2.0 \mu \mathrm{l}$ of each primer and $2.0 \mu \mathrm{l}$ of UPL probe and FastStart TaqMan Probe Master reaction mix (Roche, Mannheim, Germany). The reaction conditions were initial 
Table I. Clinicopathological characteristics of patients with prostate cancer $(n=108)$.

\begin{tabular}{lcc}
\hline Characteristics & Number of patients & $\%$ \\
\hline Age (years) & & \\
$\quad<55$ & 13 & 12.0 \\
$55-60$ & 48 & 44.5 \\
$\quad>60$ & 47 & 43.5 \\
Preoperative PSA (ng/ml) & & \\
$\quad<4.0$ & 5 & 4.6 \\
$4.0-10.0$ & 58 & 53.7 \\
$>10.0$ & 45 & 41.7 \\
Clinical stage & & \\
I & 16 & 14.8 \\
II & 63 & 58.3 \\
III & 29 & 26.9 \\
Gleason score & & \\
6 & 37 & 34.2 \\
7 & 54 & 50.0 \\
8 & 11 & 10.2 \\
9 & 6 & 5.6 \\
\hline
\end{tabular}

PSA, Prostate-specific antigen.

Table III. Relation between TMPRSS2-ERG fusion gene status and time to recurrence (Cox model).

\begin{tabular}{lcccccc}
\hline $\begin{array}{l}\text { TMPRSS2-ERG } \\
\text { status }\end{array}$ & Number of patients & HR & $95 \%$ CI & $p$-Value \\
\cline { 2 - 6 } & + & - & & & \\
\hline Mere presence & 34 & 74 & 1.52 & $0.48-4.80$ & 0.4739 \\
High expression & 13 & 95 & 6.14 & $1.95-19.39$ & $0.0020^{*}$ \\
\hline
\end{tabular}

HR, Hazard ratio; CI, confidence interval. $* p<0.05$.

denaturation at $95^{\circ} \mathrm{C}$ for $10 \mathrm{~min}$, followed by 50 cycles of $95^{\circ} \mathrm{C}$ for $10 \mathrm{~s}$ and $60^{\circ} \mathrm{C}$ for $30 \mathrm{~s}$. The expression levels were normalized to total RNA and PSA (PCA3/PSA ratio). All samples were assessed in technical duplicates. If $\mathrm{Ct}$ values obtained from technical duplicates were in discrepancy, the sample assessment was repeated.

Quantitative estimation of microRNAs. A quantitative estimation of miR-23b, miR-26a and miR-221 was performed by a RT realtime PCR method using TaqMan ${ }^{\circledR}$ MicroRNA Assays (Applied Biosystems, manufactured by Roche, Branchburg, NJ, USA). We used RNU6B (U6snRNA) as a normalizer. The Ct values were corrected using calibrators to eliminate differences between individual runs of the Stratagene Mx3005P Real-Time PCR apparatus (Agilent Technologies, Santa Clara, CA, USA). The results are presented as normalized values as a ratio of the number of copies of the given gene to that of the reference gene. To obtain gene expression data we used the $\Delta \Delta \mathrm{Ct}$ approach $\left(2^{-\Delta \Delta \mathrm{Ct}}\right.$ algorithm).
Table II. TMPRSS2-ERG fusion gene status in relationship to the stage of the disease and Gleason score.

\begin{tabular}{lcccc}
\hline TMPRSS2-ERG & \multicolumn{2}{c}{ Presence detected } & \multicolumn{2}{c}{ High expression } \\
\hline all (n=108) & 34 & $31.5 \%$ & 13 & $12.0 \%$ \\
Clinical stage & & & & \\
$\quad$ I (n=16) & 7 & $43.8 \%$ & 1 & $6.3 \%$ \\
$\quad$ II (n=63) & 13 & $20.6 \%$ & 6 & $9.5 \%$ \\
III (n=29) & 14 & $48.3 \%$ & 6 & $20.7 \%$ \\
Gleason score & & & & \\
6 (n=37) & 8 & $21.6 \%$ & 3 & $8.1 \%$ \\
$7(\mathrm{n}=54)$ & 17 & $31.5 \%$ & 7 & $13.0 \%$ \\
$8(\mathrm{n}=11)$ & 4 & $36.4 \%$ & 2 & $18.2 \%$ \\
$9(\mathrm{n}=6)$ & 5 & $83.3 \%$ & 1 & $16.7 \%$ \\
\hline
\end{tabular}

Quantitative estimation of blood serum PSA protein. Preoperative blood samples were taken from the cubital vein before any procedures involving the prostate manipulation, collected in VACUETTE $^{\circledR}$ blood collection tubes (Greiner Bio-One, Kremsmünster, Austria). The serum was separated by centrifugation at $1700 \times g$ for 10 minutes and PSA determined using the UniCel DxI 800 chemiluminescent immunoassay system (Beckman Coulter, Brea, CA, USA) as described previously (23).

Statistical analysis. SAS version 9.3 statistical software (SAS Institute Inc., Cary, NC, USA) was used for all statistical calculations. The results with $p<0.05$ were considered statistically significant. Non-parametric two-sided Wilcoxon signed-rank test was used for comparing the two groups (tumor tissue and normal prostate tissue) and Pearson's Chi-squared test for evaluating categorical data. Evaluation of prognostic significance (the relation of markers to time to recurrence) was performed as analysis of maximum likelihood estimates (Cox regression hazard model); the Kaplan-Meier survival distribution functions were generated for markers significant in Cox model.

\section{Results}

We have previously analyzed the TMPRSS2-ERG fusion gene status by the RT-qPCR method with UPL probes quantifying mRNA transcripts, so we were able to classify cases not only into categories (fusion gene present/absent) but according to the expression level as well. We detected presence of the TMPRSS2-ERG fusion in 34 out of 108 samples $(31.5 \%)$. Nevertheless, in about two thirds of positive cases it was a late amplification with cycle threshold (Ct) above 40. Therefore, we decided to distinguish a category of high level of TMPRSS2-ERG fusion transcript expression $(\mathrm{Ct} \leq 40.0)$ in which 13 out of 108 samples (12.0 \% fell within). We did not record statistically significant differences of the presence or high expression of TMPRSS2-ERG fusion gene in relationship to the stage of the disease, but there were differences related to Gleason 

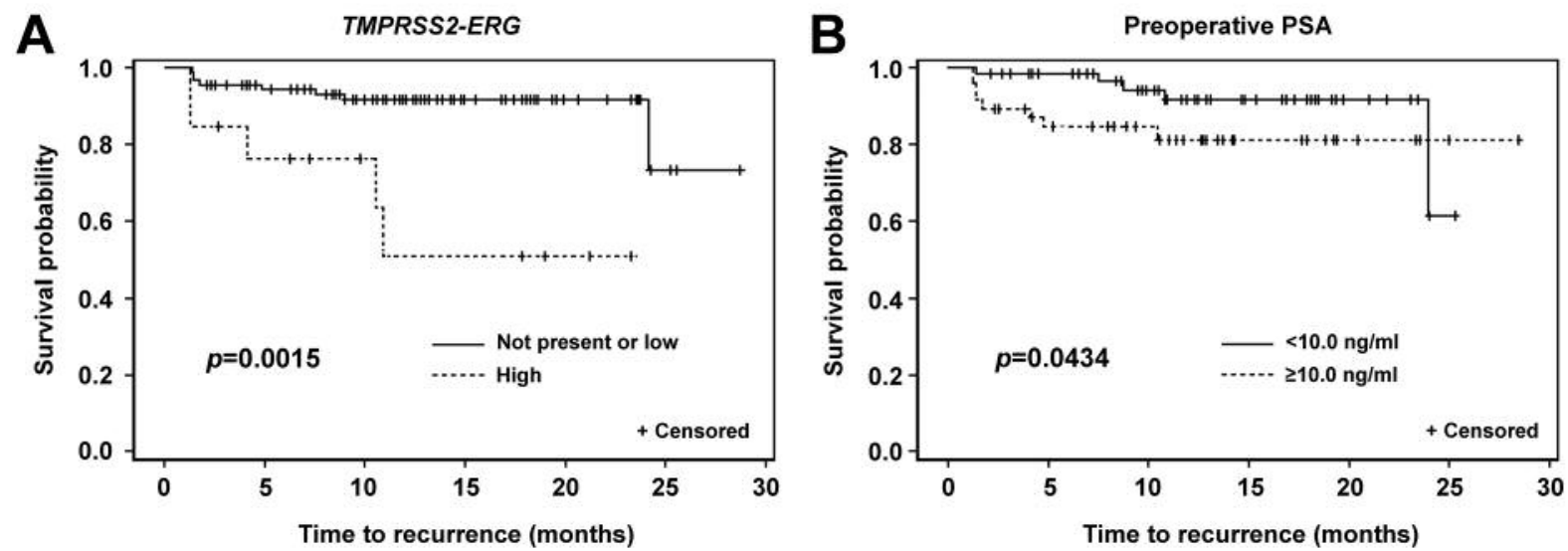

Figure 1. Relation of TMPRSS2-ERG fusion transcript expression (A) and preoperative serum PSA level (B) to time to recurrence (Kaplan-Meier curves).

TMPRSS2-ERG in combination with preoperative PSA

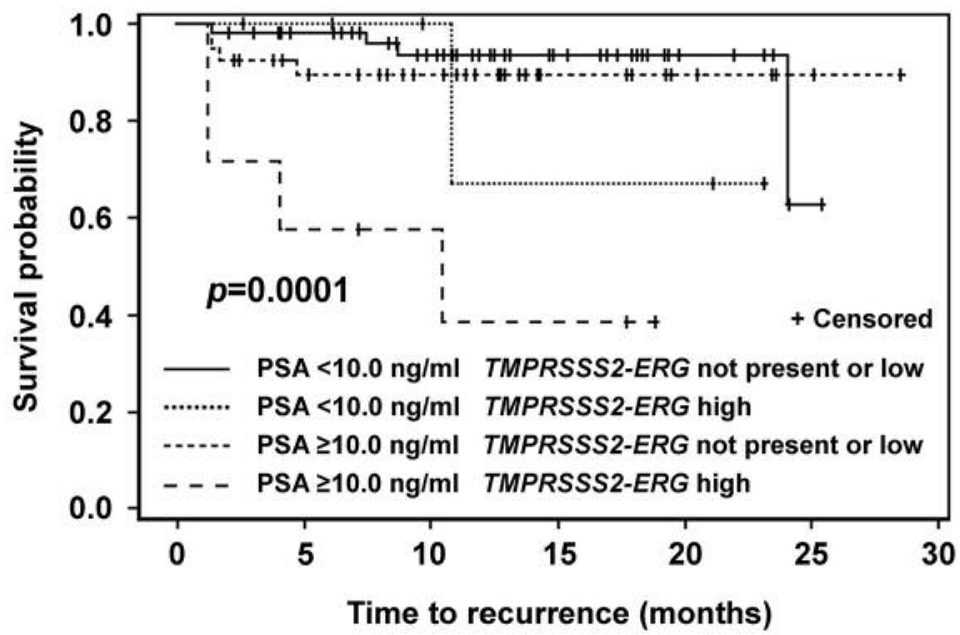

Figure 2. Relation of TMPRSS2-ERG fusion transcript expression in combination with preoperative serum PSA level to time to recurrence (KaplanMeier curves). A combination of high PSA level and high TMPRSS2-ERG expression was associated with the shortest time to recurrence.

score (Pearson's Chi-squared test, $p=0.0259$ ). Higher Gleason score was associated with higher percentage of TMPRSS2-ERG positivity (Table II).

The TMPRSS2-ERG fusion gene status was also evaluated in relation to time to recurrence (disease-free interval (DFI)). The mere presence of TMPRSS2-ERG had no relation to DFI; however, we found significantly shorter DFI in patients with high expression of TMPRSS2-ERG in the carcinoma tissue ( $p=0.0020$, Cox model, analysis of maximum likelihood estimates); details in Table III. Preoperative PSA level, the currently available biomarker for PCa, was also evaluated by Cox model and its high levels proved to be marker of shorter DFI $(p=0.0153)$. There was no correlation between preoperative PSA level and TMPRSS2-ERG fusion gene status. Kaplan-Meier survival curves were generated for both TMPRSS2-ERG fusion gene status (Figure 1A) and preoperative PSA (Figure 1B). Kaplan-Meier survival curves were also computed for the combinations of TMPRSS2-ERG status and preoperative serum PSA level (Figure 2). The category of patients with high preoperative PSA level splits according to the TMPRSS2-ERG status into groups with different prognosis; those with high expression of TMPRSS2$E R G$ have shorter DFI.

Furthermore, we evaluted the expression levels of microRNAs and found miR-23b, miR-26a and miR-221 to be down-regulated in tumor tissue compared to normal prostate 
tissue ( $p=0.0031, p=0.0022, p<0.0001$, respectively). We found no correlation of the expression of these microRNAs to clinical stage, Gleason score, TMPRSS2-ERG status or prognosis.

There was no significant difference in the expression of PSA mRNA in tumor tissue compared to normal prostate tissue. $P C A 3$ was significantly overexpressed in tumor tissue compared to normal prostate tissue for both ways of expression values normalization, to total RNA $(p<0.0001)$ and as a $P C A 3 / P S A$ ratio $(p<0.0001)$. We found no correlation of the expression of either PSA or PCA3 to clinical stage, Gleason score, TMPRSS2-ERG status or prognosis.

The expression of miR-23b, miR-26a and miR-221 had no relation to TMPRSS2-ERG status and DFI. We recorded significantly lower expression of miR-221 in tumor tissue compared to normal prostate tissue $(p=0.0005)$.

\section{Discussion}

It is known that some prostate carcinomas may not cause any trouble or only progress very slowly. Microscopic foci of prostate cancer are frequently randomly found in autopsies of men over the age of 50 who died from other reasons (24). Due to the progress in $\mathrm{PCa}$ screening, a rising number of men are diagnosed with PCa; nevertheless, not in all cases immediate radical treatment is necessary. There is an increasing need to distinguish tumors that are rapidly progressing to spare patients with slowly growing tumors the strain of overtreatment. To identify those patients with tumors that tend to progress, we used a panel of genes whose expression is significantly involved in PCa carcinogenesis (TMPRSS2-ERG, PCA3, miR23b, miR-26a and miR-221) $(12,14,25)$. We assessed their expression on RNA level that allowed their quantification using RT real time PCR. We combined these markers with serum PSA, as a routinely used marker of high senzitivity for detection of $\mathrm{PCa}$, but with a limited prognostic value.

As the most promising indicator of worse outcome, we have revealed a combination of high level of preoperative serum PSA and a presence of high level expression of TMPRSS2-ERG fusion gene in tumor tissue. Despite of limitation in specificity, serum PSA is one of the most useful biomarkers in oncology and still has not lost its potential that is evident from currently developing concept of prostate health index (Phi) (23). Therefore, the identification of new biomarkers that can be used alone or in combination with PSA is welcome to improve distinguishing the aggressive from the indolent ones (26).

The TMPRSS2-ERG fusion was identified to be the most common gene rearrangement in $\mathrm{PCa}$; published data report its prevalence approximately $50 \%$ in PSA screened localized PCa (27). In our cohort, we detected presence of the TMPRSS2-ERG fusion in $31.5 \%$ of PCa samples. The mere presence of the fusion gene in our cohort had no relation to prognosis. The meta-analysis of Pettersson et al. concluded that TMPRSS2-ERG or ERG overexpression did not strongly predict recurrence or mortality among men treated with radical prostatectomy; the cohort of this meta-analysis included patients from Europe, North America and Asia (28). It appears, however, that the levels of TMPRSS2-ERG, not merely its presence, have the dicisive effect on progression (29). Similarly, in our cohort, we found significantly shorter time to biochemical recurence in patients with high expression of TMPRSS2-ERG transcript.

TMPRSS2-ERG fusion gene presence can be detected noninvasivelly from samples of urine. Stephan et al. in their work focused on diagnostics of PCa and concluded that $P C A 3$ and Phi were superior to the other parameters and further stated that the advantage of TMPRSS2-ERG might be seen in subgroups of aggressive PCa (30).

MicroRNAs, whose expression was assessed (miR-23b, miR-26a and miR-221) in our study, were chosen on the base of described role in pathogenesis of PCa $(12,14,25)$. Nevertheless, we did not record any prognostic significance. We observed lower expression of miR-221 in PCa tumor tissue. This finding supports tumor suppressor role of miR221 in PCa, which was described in several previously published studies $(15,31,32)$. However, some other studies $(33,34)$ indicate up-regulation of miR-221 in PCa.

In conclusion, a combination of high preoperative serum PSA and high expression of TMPRSS2-ERG could be promising in distinguishing those tumors that are aggressive and together with patient status make it possible to decide if radical resection is beneficial.

\section{Conflicts of Interest}

The Authors declared they have no conflicts of interest.

\section{Acknowledgements}

This study was supported by the grant of Ministry of Health of the Czech Republic - Conceptual Development of Research Organization (Faculty Hospital in Pilsen - FNP1, 00669806) and the project of Faculty of Medicine in Pilsen SVV-2016-260283.

\section{References}

1 Attard G, Parker C, Eeles RA, Schröder F, Tomlins SA, Tannock I, Drake CG and de Bono JS: Prostate cancer. Lancet Lond Engl 387: 70-82, 2016.

2 Storås AH, Sanda MG, Boronat OG, Chang P, Patil D, Crociani C, Suarez JF, Cvancarova M, Loge JH and Fosså SD: Erectile dysfunction and sexual problems two to three years after prostatectomy among american, norwegian, and spanish patients. Clin Genitourin Cancer 14: e265-273, 2016.

3 Dahl S, Loge JH, Berge V, Dahl AA, Cvancarova M and Fosså SD: Influence of radical prostatectomy for prostate cancer on work status and working life 3 years after surgery. J Cancer Surviv Res Pract 9: 172-179, 2015. 
4 Tomlins SA, Rhodes DR, Perner S, Dhanasekaran SM, Mehra R, Sun X-W, Varambally S, Cao X, Tchinda J, Kuefer R, Lee C, Montie JE, Shah RB, Pienta KJ, Rubin MA and Chinnaiyan AM: Recurrent fusion of TMPRSS2 and ETS transcription factor genes in prostate cancer. Science 310: 644-648, 2005.

5 Rubin MA and Chinnaiyan AM: Bioinformatics approach leads to the discovery of the TMPRSS2:ETS gene fusion in prostate cancer. Lab Investig J Tech Methods Pathol 86: 1099-1102, 2006.

6 Shafi AA, Yen AE and Weigel NL: Androgen receptors in hormone-dependent and castration-resistant prostate cancer. Pharmacol Ther 140: 223-238, 2013.

7 Cai C, Wang H, Xu Y, Chen S and Balk SP: Reactivation of androgen receptor-regulated TMPRSS2:ERG gene expression in castration-resistant prostate cancer. Cancer Res 69: 6027-6032, 2009.

8 Carver BS, Tran J, Gopalan A, Chen Z, Shaikh S, Carracedo A, Alimonti A, Nardella C, Varmeh S, Scardino PT, Cordon-Cardo C, Gerald W and Pandolfi PP: Aberrant ERG expression cooperates with loss of PTEN to promote cancer progression in the prostate. Nat Genet 41: 619-624, 2009.

9 Hagan JP and Croce CM: MicroRNAs in carcinogenesis. Cytogenet Genome Res 118: 252-259, 2007.

10 Bertoli G, Cava C and Castiglioni I: MicroRNAs as Biomarkers for Diagnosis, Prognosis and Theranostics in Prostate Cancer. Int J Mol Sci 17, 2016.

11 Goto Y, Kojima S, Nishikawa R, Enokida H, Chiyomaru T, Kinoshita T, Nakagawa M, Naya Y, Ichikawa T and Seki N: The microRNA-23b/27b/24-1 cluster is a disease progression marker and tumor suppressor in prostate cancer. Oncotarget 5: 77487759, 2014.

12 Majid S, Dar AA, Saini S, Arora S, Shahryari V, Zaman MS, Chang I, Yamamura S, Tanaka Y, Deng G and Dahiya R: miR-23b represses proto-oncogene Src kinase and functions as methylationsilenced tumor suppressor with diagnostic and prognostic significance in prostate cancer. Cancer Res 72: 6435-6446, 2012.

13 Zhao S, Ye X, Xiao L, Lian X, Feng Y, Li F and Li L: MiR-26a inhibits prostate cancer progression by repression of Wnt5a. Tumour Biol J Int Soc Oncodevelopmental Biol Med 35: 9725 9733, 2014.

14 Kato M, Goto Y, Matsushita R, Kurozumi A, Fukumoto I, Nishikawa R, Sakamoto S, Enokida H, Nakagawa M, Ichikawa $\mathrm{T}$ and Seki N: MicroRNA-26a/b directly regulate La-related protein 1 and inhibit cancer cell invasion in prostate cancer. Int J Oncol 47: 710-718, 2015.

15 Zheng Q, Peskoe SB, Ribas J, Rafiqi F, Kudrolli T, Meeker AK, De Marzo AM, Platz EA and Lupold SE: Investigation of miR21, miR-141, and miR-221 expression levels in prostate adenocarcinoma for associated risk of recurrence after radical prostatectomy. The Prostate 74: 1655-1662, 2014.

16 Cui Y, Cao W, Li Q, Shen H, Liu C, Deng J, Xu J and Shao Q: Evaluation of prostate cancer antigen 3 for detecting prostate cancer: a systematic review and meta-analysis. Sci Rep 6: 25776, 2016.

17 Ferro M, Lucarelli G, Bruzzese D, Perdonà S, Mazzarella C, Perruolo G, Marino A, Cosimato V, Giorgio E, Tagliamonte V, Bottero D, De Cobelli $O$ and Terracciano D: Improving the prediction of pathologic outcomes in patients undergoing radical prostatectomy: the value of prostate cancer antigen 3 (PCA3), prostate health index (phi) and sarcosine. Anticancer Res 35: 1017-1023, 2015
18 Cantiello F, Russo GI, Ferro M, Cicione A, Cimino S, Favilla V, Perdonà $\mathrm{S}$, Bottero $\mathrm{D}$, Terracciano $\mathrm{D}$, De Cobelli $\mathrm{O}$, Morgia $\mathrm{G}$ and Damiano R: Prognostic accuracy of Prostate Health Index and urinary Prostate Cancer Antigen 3 in predicting pathologic features after radical prostatectomy. Urol Oncol 33: 163.e15-23, 2015.

19 Kalfert D, Pesta M, Kulda V, Topolcan O, Ryska A, Celakovsky $\mathrm{P}$, Laco $\mathrm{J}$ and Ludvikova M: MicroRNA profile in site-specific head and neck squamous cell cancer. Anticancer Res 35: 24552463, 2015

20 Smid D, Kulda V, Srbecka K, Kubackova D, Dolezal J, Daum O, Kucera R, Topolcan O, Treska V, Skalicky T and Pesta M: Tissue microRNAs as predictive markers for gastric cancer patients undergoing palliative chemotherapy. Int J Oncol 48: 2693-2703, 2016.

21 Kan Z, Jaiswal BS, Stinson J, Janakiraman V, Bhatt D, Stern HM, Yue P, Haverty PM, Bourgon R, Zheng J, Moorhead M, Chaudhuri S, Tomsho LP, Peters BA, Pujara K, Cordes S, Davis DP, Carlton VEH, Yuan W, Li L, Wang W, Eigenbrot C, Kaminker JS, Eberhard DA, Waring P, Schuster SC, Modrusan Z, Zhang Z, Stokoe D, de Sauvage FJ, Faham M and Seshagiri S: Diverse somatic mutation patterns and pathway alterations in human cancers. Nature 466: 869-873, 2010.

22 Danila DC, Anand A, Sung CC, Heller G, Leversha MA, Cao L, Lilja H, Molina A, Sawyers CL, Fleisher M and Scher HI: TMPRSS2-ERG status in circulating tumor cells as a predictive biomarker of sensitivity in castration-resistant prostate cancer patients treated with abiraterone acetate. Eur Urol 60: 897-904, 2011.

23 Fuchsova R, Topolcan O, Windrichova J, Hora M, Dolejsova O, Pecen L, Kasik P, Novak J, Casova M and Smejkal J: PHI in the early detection of prostate cancer. Anticancer Res 35: 4855$4857,2015$.

24 Stangelberger A, Waldert M and Djavan B: Prostate cancer in elderly men. Rev Urol 10: 111-119, 2008.

25 Kneitz B, Krebs M, Kalogirou C, Schubert M, Joniau S, van Poppel H, Lerut E, Kneitz S, Scholz CJ, Ströbel P, Gessler M, Riedmiller H and Spahn M: Survival in patients with high-risk prostate cancer is predicted by miR-221, which regulates proliferation, apoptosis, and invasion of prostate cancer cells by inhibiting IRF2 and SOCS3. Cancer Res 74: 2591-2603, 2014.

26 Sanguedolce F, Cormio A, Brunelli M, D’Amuri A, Carrieri G, Bufo P and Cormio L: Urine TMPRSS2: ERG fusion transcript as a biomarker for prostate cancer: Literature Review. Clin Genitourin Cancer 14: 117-121, 2016.

27 Tomlins SA, Bjartell A, Chinnaiyan AM, Jenster G, Nam RK, Rubin MA and Schalken JA: ETS gene fusions in prostate cancer: from discovery to daily clinical practice. Eur Urol 56 : 275-286, 2009.

28 Pettersson A, Graff RE, Bauer SR, Pitt MJ, Lis RT, Stack EC, Martin NE, Kunz L, Penney KL, Ligon AH, Suppan C, Flavin R, Sesso HD, Rider JR, Sweeney C, Stampfer MJ, Fiorentino M, Kantoff PW, Sanda MG, Giovannucci EL, Ding EL, Loda M and Mucci LA: The TMPRSS2:ERG rearrangement, ERG expression, and prostate cancer outcomes: a cohort study and meta-analysis. Cancer Epidemiol Biomark Prev Publ Am Assoc Cancer Res Cosponsored Am Soc Prev Oncol 21: 1497-1509, 2012.

29 Font-Tello A, Juanpere N, de Muga S, Lorenzo M, Lorente JA, Fumado L, Serrano L, Serrano S, Lloreta J and Hernández S: Association of ERG and TMPRSS2-ERG with grade, stage, and prognosis of prostate cancer is dependent on their expression levels. The Prostate 75: 1216-1226, 2015. 
30 Stephan C, Jung K, Semjonow A, Schulze-Forster K, Cammann $\mathrm{H}, \mathrm{Hu} \mathrm{X}$, Meyer H-A, Bögemann M, Miller K and Friedersdorff F: Comparative assessment of urinary prostate cancer antigen 3 and TMPRSS2:ERG gene fusion with the serum [-2]proprostatespecific antigen-based prostate health index for detection of prostate cancer. Clin Chem 59: 280-288, 2013.

31 Guzel E, Karatas OF, Semercioz A, Ekici S, Aykan S, Yentur S, Creighton CJ, Ittmann $\mathrm{M}$ and Ozen $\mathrm{M}$ : Identification of microRNAs differentially expressed in prostatic secretions of patients with prostate cancer. Int J Cancer 136: 875-879, 2015.

32 Xuan H, Xue W, Pan J, Sha J, Dong B and Huang Y: Downregulation of miR-221, -30d, and -15 a contributes to pathogenesis of prostate cancer by targeting Bmi-1. Biochemistry (Mosc) 80: 276-283, 2015.
33 Sun T, Yang M, Chen S, Balk S, Pomerantz M, Hsieh C-L, Brown M, Lee G-SM and Kantoff PW: The altered expression of MiR-221/-222 and MiR-23b/-27b is associated with the development of human castration resistant prostate cancer. The Prostate 72: 1093-1103, 2012.

34 Song $\mathrm{C}$, Chen $\mathrm{H}$, Wang $\mathrm{T}$, Zhang $\mathrm{W}, \mathrm{Ru} \mathrm{G}$ and Lang $\mathrm{J}$ : Expression profile analysis of microRNAs in prostate cancer by next-generation sequencing. The Prostate 75: 500-516, 2015.

Received July 1, 2016

Revised August 17, 2016

Accepted August 18, 2016 